\title{
La culture du coton ne bouleverse pas les échanges traditionnels de semences de sorgho
}

\author{
Sandra Delaunay ${ }^{1}$ \\ Robers-Pierre Tescar ${ }^{2}$ \\ Auguste Oualbego ${ }^{3}$ \\ Kirsten vom Brocke ${ }^{4,5}$ \\ Jacques Lançon ${ }^{2,6}$ \\ ${ }^{1}$ Le Haut-Pont 35120 France \\ ${ }^{2}$ Centre de coopération internationale \\ en recherche agronomique \\ pour le développement (Cirad) \\ Unité propre de recherche (UPR) \\ "Systèmes de culture annuels ", \\ Avenue Agropolis, \\ TA B-102-2, \\ Montpellier 34398 cedex 5 \\ <jacques.lancon@cirad.fr> \\ ${ }^{3}$ Institut de l'environnement \\ et de recherches agricoles (Inera), \\ Centre régional de recherche du Centre, \\ Station de Saria, \\ BP 10, \\ Koudougou \\ Burkina Faso \\ ${ }^{4}$ Institut de l'environnement \\ et de recherches agricoles (Inera), \\ 01 BP 596 , \\ Ouagadougou \\ Burkina-Faso \\ ${ }^{5}$ Centre de coopération internationale \\ en recherche agronomique \\ pour le développement (Cirad), \\ Unité propre de recherche (UPR) \\ "Agrobiodiversité des plantes de savanes ", \\ Avenue Agropolis \\ TA A-08/01, \\ 34398 Montpellier cedex 5 \\ France \\ <kirsten.vom_brocke@cirad.fr> \\ ${ }^{6}$ Institut national des recherches agricoles \\ du Bénin (Inrab), \\ Direction générale, \\ 01 BP 884 \\ Cotonou Bénin \\ <jacques.lancon@cirad.fr>
}

Tirés à part : J. Lançon

\begin{abstract}
Résumé
La plupart des cultivateurs de sorgho produisent leurs propres semences ou les obtiennent par échange. Ils utilisent peu les variétés proposées par les systèmes semenciers formels. C'est que l'échange de semences renforce les réseaux traditionnels de solidarité et favorise une gestion collective des risques, dans des conditions de culture particulièrement précaires. Notre étude vise à caractériser ces échanges et à vérifier si leur fréquence ou leurs modalités peuvent être modifiées dans un village produisant une culture commerciale, en l'occurrence, du coton. Elle a été conduite en 2004 et 2005 dans cinq villages du Burkina Faso. Les agriculteurs enquêtés ont été échantillonnés suivant la méthode "boule de neige ", qui permet de reconstituer plus facilement l'ensemble des liens d'échange. Les résultats montrent que les agriculteurs achètent et vendent peu de semences lorsque leurs récoltes sont suffisantes. La plupart des mouvements de semences sont gratuits ou non marchands, et ils tendent à suivre des règles traditionnelles. Plus les individus sont proches géographiquement et socialement, plus les échanges sont nombreux. Ainsi, l'échange a le plus souvent lieu entre personnes de même village, de même ethnie, ou de même famille. Néanmoins, ces pratiques ne sont pas exclusives et elles coexistent avec des échanges entre personnes non apparentées et de villages éloignés. En 2005, la comparaison d'un village "coton" (Siby) avec un village sans coton (Tiogo) a montré que les producteurs de coton se comportent comme les autres agriculteurs. Ils échangent autant de semences et de la même manière. Les réseaux informels d'échanges ne semblent donc pas menacés par le coton. Ceux du village cotonnier sont moins fournis mais plus étendus. Le don et les échanges intra-ethniques restent privilégiés et les échanges marchands n'ont pas augmenté. En revanche, l'organisation sociale engendrée par le coton a favorisé le développement d'échanges nouveaux, entre amis, alors que, dans le village sans coton, les échanges sont restés essentiellement familiaux. Grâce au coton, certains agriculteurs peuvent thésauriser et accéder à un mode alternatif et individuel de gestion de risque, mais ils restent néanmoins impliqués dans le système traditionnel tout en intégrant de nouveaux membres dans leurs échanges.
\end{abstract}

Mots clés : coton; réseau ; semence ; sorgho.

Thèmes : économie et développement rural ; productions végétales.

\section{Abstract}

\section{Growing cotton does not disrupt traditional sorghum seed exchange}

Most sorghum growers either produce their own seed or obtain it through exchange. They seldom use seed delivered by formal seed systems. The reason is that seed exchange strengthens traditional solidarity networks which are the basis for collective risk management in the case of precarious cropping conditions. Our study aimed at characterizing the seed exchange system in sorghum; and verifying if frequency or modalities could be modified in a village where farmers are used to making money from cotton growing. It was carried out in 2004 and 2005, in five villages in Burkina Faso. Farmers were sampled according to the "snowball" method. The results show that farmers usually buy and sell small quantities of seed when their harvests are sufficient. In general, seeds are given for free and according to traditional rules. People exchange more with people who are close to them geographicaly or socially, and such exchanges mostly take place within villages, ethnic groups, or families. However, such rules are not exclusive, and exchanges also happen among people who are unrelated or from remote villages. In 2005, the comparison between two villages, one growing cotton (Siby) and the other not (Tiogo), showed that the cotton producers behaved like the others. They exchange as many seeds and in the same way. Cotton does not seem to modify the traditional exchange network. 
Gift and intra-ethnic exchanges remain frequent and trade exchanges do not increase. On the other hand, the social organization generated by cotton production eased the development of new exchanges, among friends, while in the non-cotton producing village, the exchanges were essentially kept within the family. By growing cotton, farmers create a source of income and an access to an alternative and individual mode of risk management. While integrating new members into their exchanges, they also remain involved in the traditional system.

Key words: cotton; networks; seed; sorghum grain.

Subjects: economy and rural development; vegetal productions.

a majorité des producteurs de cultures vivrières produisent eux-mêmes leur semence ou l'obtiennent par échange. Ils utilisent peu les variétés améliorées pour des raisons de disponibilité, d'accès ou d'inadaptation aux conditions locales (Remington et al., 2002). L'enjeu pour les chercheurs est donc de comprendre les règles de l'échange pour améliorer les performances du système et permettre aux agriculteurs de disposer de semences en quantité suffisante, en temps opportun et de qualité physiologique ou génétique adéquate.

Pour Almekinders (2001), les échanges de semences existant au sein des communautés forment un "système " semencier, qualifié d'informel, de traditionnel ou de local. Ces systèmes se caractérisent par le réseau formé par les flux de semences échangées, mais aussi par les règles et les pratiques d'échange. Une meilleure connaissance de ces échanges informels est utile pour caractériser les flux génétiques entre variétés locales et concevoir des aménagements propices à leur conservation ou à la diffusion des variétés améliorées.

Le don, le troc, les transactions sur les marchés villageois constituent les principaux modes d'échange et de distribution des semences au sein des réseaux informels (Almekinders et al. 1994 ; Tripp, 2001). Certains de ces modes reposent sur des obligations sociales qui surdéterminent les stratégies des acteurs.

Le don se définit comme l'action de céder gratuitement quelque chose à quelqu'un. Encadrés par des codes sociaux et moraux, dons et contre-dons renforcent la solidarité au sein des communautés proches (Mauss, 1923-1924). Le troc est un échange sans médiation fiduciaire, un don immédiatement annulé par un contre-don. Selon Badstue et al. (2002), le troc franchit plus facilement les barrières sociales. Enfin, les transactions sur les marchés sont médiées par de l'argent, et les agriculteurs préfèrent acheter leurs semences sur les marchés locaux, car les marchands peu scrupuleux y sont rapidement mis à l'index.

Les échanges informels de semences obéissent-ils à une logique fondée sur des règles collectives ou des tactiques individuelles? Badstue et al. (2002) ou Van Dusen (2004) au Mexique, Nagarajan et Smale (2005) en Inde ont constaté que les échanges avec les voisins proches sont importants, tout en restant modulés par les affinités personnelles. Néanmoins, la majorité des échanges de semences se réalisent entre les membres d'une même famille, la définition de l'apparentement pouvant toutefois varier entre cultures ou entre ethnies. Il existe ainsi, dans la tradition mexicaine, des relations fortes et formelles avec le compadre qui ne fait pas partie de la famille tout en étant respecté comme un père. En revanche, les simples connaissances et les inconnus interviennent très peu dans les réseaux semenciers traditionnels (Badstue et al., 2002).

L'étude que nous avons conduite dans cinq villages du Burkina Faso en 2004 et 2005, cherchait à reconstituer la logique des échanges de semences d'une culture traditionnelle, le sorgho. Plus particulièrement, notre travail visait à vérifier que le comportement des agriculteurs dans l'échange est induit par des critères de proximité géographique ou sociale, familiale ou ethnique, et que ce comportement est modifié par la culture du coton. On pouvait en effet penser que les agriculteurs risquent de se désolidariser du système traditionnel d'échange lorsqu'ils ont la possibilité d'épargner grâce à la vente du coton et qu'ils peuvent miser sur de nouvelles solidarités au sein des réseaux professionnels créés par la production cotonnière.

\section{Méthode}

\section{Milieu d'étude}

Trois villages du Burkina Faso en 2004 et deux en 2005 ont été enquêtés. Il s'agit de Raguitenga, Vélia, et Kéra en 2004, et de Tiogo et Siby en 2005, dont les situations géographiques sont indiquées sur la figure 1.

Leurs caractéristiques géographiques et ethniques sont indiquées au tableau 1. Seuls Kéra et Siby produisent du coton. Les situations climatiques des deux années sont comparables. La faible pluviométrie et l'irrégularité des pluies au moment des semis ont entraîné des récoltes irrégulières et particulièrement insuffisantes en 2004 à Tiogo et Siby suite à deux années successives peu favorables au moment des semis. Dans ces cinq villages, les exploitants cultivent à la fois du sorgho rouge plutôt réservé à la production de boisson alcoolisée, le dolo, et de couscous et du sorgho blanc, souvent préféré pour la fabrication du $t \hat{o}^{1}$. Mais les pratiques évoluent et il n'est pas rare que les sorghos rouges ou blancs soient utilisés indifféremment suivant l'état des réserves. Nous avons caractérisé le niveau moyen d'autonomie des exploitations de chaque village au moyen de deux indicateurs, le ratio d'autonomie alimentaire (RAA) et le ratio d'autonomie semencière (RAS) :

- RAA = grain consommé/(grain produit ou acheté) ;

- RAS $=$ semences produites et semées/ semences semées.

Pour classer les bénéficiaires des relations d'échange, nous avons dû adopter une définition des notions de famille, ami, parenté (encadré 1).

\footnotetext{
tô : bouillie traditionnelle préparée quotidiennement en Afrique de l'Ouest à partir d'une farine de sorgho, de mil ou de maïs.
} 


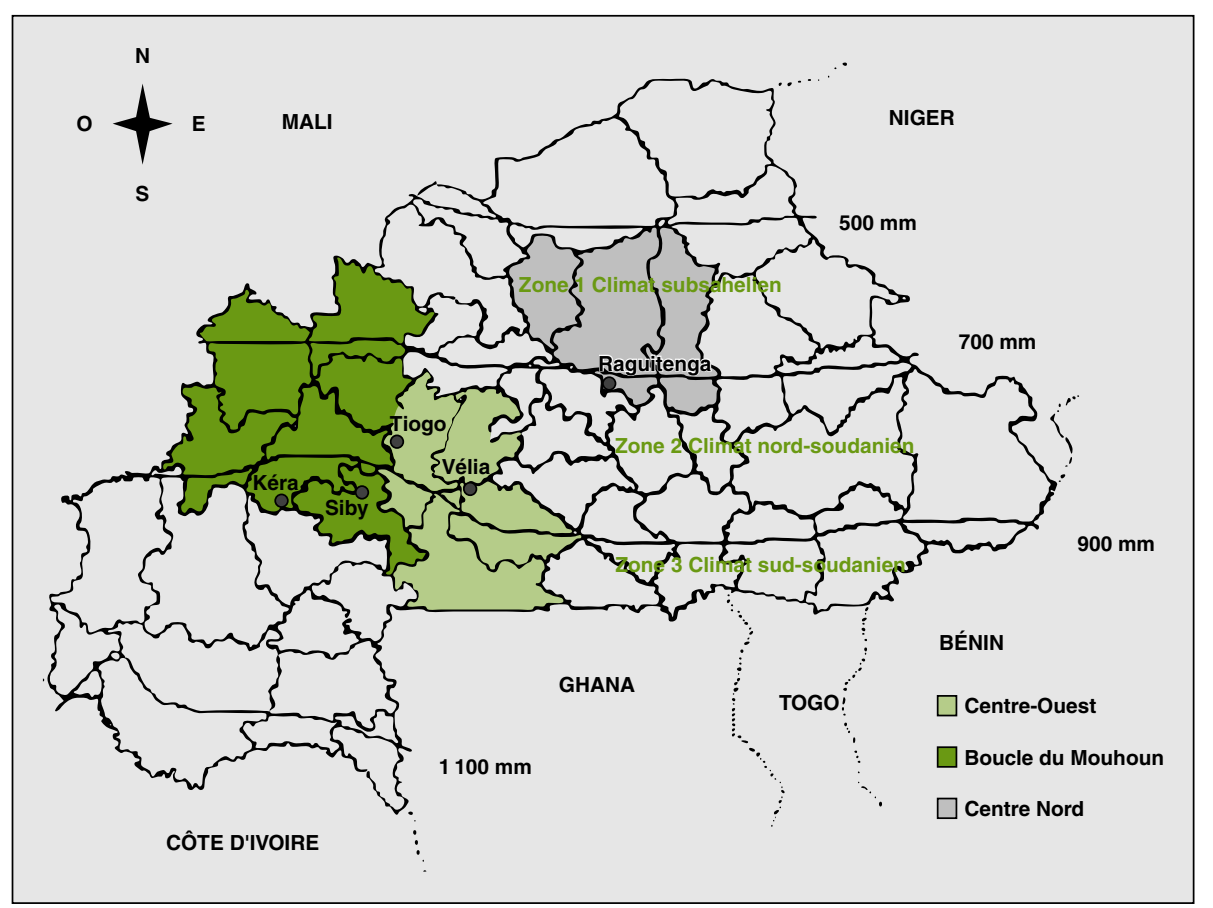

Figure 1. Localisation des cinq villages d'étude.

Figure 1. Location of the five selected villages.

\section{Encadré 1 \\ Définitions des notions utilisées pour la détermination des types de relation sociale entre les enquêtés}

Famille et parenté : la famille ne se restreint pas au groupe de personnes qui vivent ensemble autour du même foyer (parents, enfants, voire grandparents). Elle inclut les gens liés par le sang (enfants, oncles, tantes, cousins...) et les alliés (beau-père, belle-fille...).

Ami : personne proche du chef d'exploitation (CE), sans lien de sang ou d'alliance avec lui, mais considérée comme membre actif socialement pour le groupe familial (services rendus, relations politiques, participation aux événements importants dans la famille, etc.)

Connaissance : personne connue mais avec qui le CE n'a pas de relation sociale suivie.

Non apparenté : celui qui n'est lié au CE ni par le sang ni par l'alliance.

\section{Technique d'échantillonnage et traitement des données}

Les chefs d'exploitation (CE) ont été échantillonnés suivant la méthode dite "boule de neige". Utilisée par Subedi et al (2003) pour étudier la structure des réseaux informels de semences de riz au Népal, cette méthode consiste à identifier et suivre le plus loin possible (en pratique, jusqu'au sixième niveau) l'ensemble des liens d'échanges à partir des déclarations d'un CE pris au hasard dans la population et considéré comme le meneur de chaînes. Les CE qui ont échangé du grain ou des semences de sorgho avec lui appartiennent au rang 2 et ainsi de suite. Une chaîne s'interrompt lorsque les échanges mentionnés renvoient à des CE déjà pris en compte. Les CE identifiés au niveau $\mathrm{n}$ déterminent donc par leurs échanges les CE du niveau $\mathrm{n}+1$.

Les enquêtes ont porté principalement sur les échanges de semences, mais en 2005, à Siby et Tiogo, nous avons aussi tenu compte des échanges de grains.

Dans les villages de 2004 et dans celui de Tiogo en 2005, l'échantillonnage de départ a été réalisé avec le conseiller sorgho de la zone, qui a proposé trois meneurs de chaîne par village, choisis dans trois quartiers différents. Les meneurs se différenciaient aussi entre eux pour la détention des moyens de production (animaux de trait, charrues), l'appartenance ethnique, l'âge et la confession religieuse. Dans le village coton de Siby, en 2005, les meneurs de chaîne ont été tirés au hasard dans 3 groupements de producteurs de coton (GPC) parmi les 7 que compte le village. Au total, $89 \mathrm{CE}$ ont été enquêtés en 2004 et 99 en 2005: 27 à Vélia, 30 à Raguitenga, 32 à Kéra, 66 à Tiogo et 33 à Siby.

Tableau 1. Caractérisation des cinq villages d'enquête.

Table 1. Characterisation of the five selected villages.

\begin{tabular}{|c|c|c|c|c|c|}
\hline \multirow{2}{*}{$\begin{array}{c}\text { Année d'enquête } \\
\text { Village }\end{array}$} & \multicolumn{3}{|c|}{2004} & \multicolumn{2}{|c|}{2005} \\
\hline & Vélia & Raguitenga & Kéra & Tiogo & Siby \\
\hline Région & Centre-Ouest & Centre-Nord & Boucle Mouhoun & Centre-Ouest & Boucle Mouhoun \\
\hline Province & Ziro & Sanmatenga & Mouhoun & Sanguié & Les Balés \\
\hline Nombre d'habitants & 1367 & 370 & 1811 & 2864 & 3723 \\
\hline Ethnies interrogées & Mossi & Mossi & Bwaba, Mossi & $\begin{array}{l}\text { Mossi, } \\
\text { Gourounsi }\end{array}$ & Kô, Mossi \\
\hline $\begin{array}{l}\text { Présence } \\
\text { de la culture de coton }\end{array}$ & Non & Non & Oui & Non & Oui \\
\hline
\end{tabular}


Pour évaluer l'effet de la culture du coton en 2005 dans les villages de Siby et Tiogo, nous avons comparé les nombres rie par une analyse de Kruskal-Wallis (Anova par rang) car le test de Kolmogorov et Smirnov montrait que les distributions n'étaient pas normales.

\section{Résultats}

Quelques informations sur les CE enquêtés sont réunies au tableau 2. Leur âge moyen est comparable dans les différents villages, même s'ils paraissent un peu plus jeunes dans les villages cotonniers. En 2005, le taux d'équipement en charrues était nettement plus important dans le village cotonnier de Siby, la coutume moyens d'échanges recensés par catégo-

voulant qu'à Tiogo l'exploitant offre un animal de trait en sacrifice à ses ancêtres avant de devenir propriétaire de son propre animal. Les surfaces en sorgho étaient un peu plus importantes dans le village non cotonnier de Tiogo pour un ratio d'autosuffisance en semences et en grains inférieur à celui de Siby.

Dans les villages étudiés, les semences de sorgho sont fréquemment données ou troquées. En 2005, environ $25 \%$ des CE de Tiogo et Siby ont déclaré avoir donné des semences, autant en ont reçu et 1 sur 10 en a troqué. C'est un peu moins qu'en 2004, où la moitié des CE de Kera, Raguitenga et Velia, ont déclaré en avoir donné ou troqué. Pour les CE interviewés, le don de semences est un acte de solidarité que l'on ne peut pas refuser à un proche, et qu'on fait spontanément lorsque celui-ci est dans le besoin. En revanche, les fournisseurs de semences admettent difficile-

\section{Tableau 2. Caractéristiques de l'échantillon de chefs d'exploitations (CE) interrogés dans les cinq villages de l'étude.}

Table 2. Characteristics of the sample of farmers interviewed in the five selected villages.

\begin{tabular}{lccccc}
\hline \multicolumn{1}{c}{\begin{tabular}{c} 
Année d'enquête \\
\multicolumn{1}{c}{ Village }
\end{tabular}} & \multicolumn{3}{c}{$\mathbf{2 0 0 4}$} & \multicolumn{2}{c}{$\mathbf{2 0 0 5}$} \\
\cline { 2 - 6 } & Vélia & Raguitenga & Kéra & Tiogo & Siby \\
\hline $\begin{array}{l}\text { Âge moyen des CE } \\
\text { (erreur standard) }\end{array}$ & 48 & 48 & 42 & 50 & 45 \\
\hline $\begin{array}{l}\text { Niveau d'équipement } \\
\text { \% CE avec charrue à } \\
\text { traction animale) }\end{array}$ & $\mathrm{ND}$ & $\mathrm{ND}$ & $\mathrm{ND}$ & 11 & 64 \\
\hline $\begin{array}{l}\text { Surface sorgho moyenne } \\
\text { constatée - en ha/CE }\end{array}$ & 2,6 & 2,7 & 2,6 & 3,3 & 2,3 \\
(erreur standard) & $(0,27)$ & $(0,31)$ & $(0,28)$ & $(0,26)$ & $(0,24)$ \\
\hline RAS & & & & & \\
RAA & 0,83 & 0,99 & 0,70 & 0,81 & 0,91 \\
& 1,00 & 1,00 & 1,00 & 0,82 & 0,90 \\
\hline
\end{tabular}

RAS : ratio d'autosuffisance en semences (constaté) ; RAA : ratio d'autosuffisance alimentaire (constaté) ; ND : non déterminé.

Tableau 3. Quantités (kg/CE) et origine des graines de sorgho effectivement utilisées comme semences dans les exploitations des cinq villages d'enquête.

Table 3. Quantities (kg/farmer) and origin of the sorghum seeds actually sown in the farms of the five villages.

\begin{tabular}{lrcccrr}
\hline \multirow{2}{*}{ Année } & \multicolumn{3}{c}{$\mathbf{2 0 0 4}$} & & \multicolumn{2}{c}{$\mathbf{2 0 0 5}$} \\
\cline { 2 - 3 } \cline { 6 - 7 } Origine des semences & Vélia & Raguitenga & Kéra & & Tiogo & Siby \\
\hline Autoproduites & 15,8 & 15,8 & 5,9 & & 23,5 & 19,2 \\
Dons & 3,8 & 0,5 & 2,1 & & 2,8 & 2,1 \\
Trocs & 1,1 & 1,0 & 0,5 & & 1,2 & 0,3 \\
Achats & 0,0 & 0,0 & 1,4 & & 2,4 & 0,3 \\
Total échangé & 4,9 & 1,5 & 4 & & 6,4 & 2,7 \\
Total semé & 20,7 & 17,3 & 9,9 & & 29,9 & 21,9 \\
\hline
\end{tabular}

ment en avoir reçu. Dans le besoin, ils préféreront troquer avec une personne réputée comme eux pour ses semences.

Dans les quatre villages de Vélia, Kéra, Tiogo et Siby, les quantités données dépassent celles troquées et achetées (tableau 3). En revanche, les agriculteurs de Raguitengua ont troqué deux fois plus qu'ils n'ont donné. À Kera et Tiogo, les achats sont plus importants que dans les autres villages et ils représentent autant que les trocs. On remarquera que les quantités de semences utilisées à Kéra sont particulièrement faibles et qu'elles ne permettent théoriquement pas de semer l'ensemble des surfaces déclarées.

Lorsque leurs récoltes sont suffisantes, les agriculteurs achètent et vendent peu. Partout, les CE ont déclaré ne s'approvisionner sur le marché qu'en dernier recours et dans deux cas: si les récoltes ont été mauvaises, chez eux et dans leur entourage, ou s'ils recherchent des variétés qui ne sont pas cultivées par leurs proches. En 2005, leur autosuffisance semencière ou alimentaire étant meilleure qu'à Tiogo, les CE de Siby se sont peu approvisionnés sur le marché, et lorsqu'ils l'ont fait, c'était surtout pour obtenir des variétés nouvelles. En revanche, à Tiogo, les CE ont pratiquement autant acheté que donné. En situation déficitaire, les faibles réserves des donneurs n'ont pas suffi et les demandeurs ont dû recourir au marché. Dans ce village sans coton, les CE disent vendre facilement, mais seulement si leurs récoltes sont largement excédentaires car il leur faut réserver une partie pour leurs proches.

Les stratégies individuelles paraissent influencées par les relations de proximité géographique et sociale. En dehors de Raguitenga où la moitié des échanges se sont faits hors du village en 2004, les CE échangent préférentiellement avec des personnes de leur village et même de leur quartier (tableau 4). À Kera comme à Siby et Tiogo, la majorité des semences circulent à l'intérieur des quartiers. Toutefois, les réseaux d'échange restent assez largement ouverts sur les autres quartiers et d'autres villages qui peuvent être distants de plus de $100 \mathrm{~km}$. La proximité sociale détermine également les stratégies d'échange. La famille est privilégiée : elle représente $60 \%$ des échanges réalisés dans les cinq villages. Elle facilite même les liens externes au village puisqu'on échange des semences en se rendant visite. Mais dans les villages de Vélia, Kéra et Siby, les CE échangent aussi régulièrement avec des personnes qui ne 
Tableau 4. Répartition des échanges en fonction de critères géographiques et sociaux dans les cinq villages (en \% du nombre total d'échanges déclarés par les interviewés dans le village).

Table 4. Exchanges according to social and geographical criteria in the five selected villages (in \% of the total rate of exchange interviewees).

\begin{tabular}{lcccccc}
\hline & \multicolumn{3}{c}{$\mathbf{2 0 0 4}$} & \multicolumn{2}{c}{$\mathbf{2 0 0 5}$} & \multirow{2}{*}{ Ensemble } \\
\cline { 2 - 6 } & Vélia & Raguitenga & Kéra & Tiogo & Siby & \\
\hline Intra-quartier & 37 & 22 & 52 & 71 & 68 & 50 \\
Hors quartier & 26 & 28 & 20 & 16 & 17 & 21 \\
Hors village & 37 & 50 & 28 & 13 & 15 & 29 \\
Parent & 55 & 83 & 58 & 61 & 44 & 60 \\
Ami/connaissance & 45 & 17 & 42 & 39 & 56 & 40 \\
Même ethnie & ND & ND & 95 & 90 & 88 & 91 \\
Ethnie différente & ND & ND & 5 & 10 & 12 & 9 \\
\hline
\end{tabular}

ND : non déterminé leur sont pas apparentées et qu'ils qualifient d'amis ou de connaissances. Dans habitent, plus de $90 \%$ des échanges de semences se font entre personnes de même ethnie.

La présence du coton dans les exploitations perturbe peu les stratégies d'échange (tableau 5). Les producteurs de coton tendent néanmoins à donner plus souvent, tant à l'extérieur du village qu'aux amis. En revanche, ils recourent les villages où plusieurs ethnies co- moins au marché et au troc que les producteurs non cotonniers.

\section{Discussion}

Cette étude confirme d'abord qu'une grande majorité des semences de sorgho, 70 à $99 \%$ suivant les villages et les années, est produite sur l'exploitation

\section{Tableau 5. Relation entre culture du coton, nombre et structure géographique et sociale des échanges chez 99 chefs d'exploitation (CE) dans les deux villages de Siby et Tiogo en 2005 (nombre moyen par CE).}

Table 5. Links between cotton-growing, number and social/geographical structure in exchanges in 2005 (average number of exchanges among 99 farmers in the villages of Tiogo and Siby).

\begin{tabular}{llcc}
\hline & Intra-quartier & $\begin{array}{c}\text { Coton } \\
\text { (24 CE) }\end{array}$ & $\begin{array}{c}\text { Sans coton } \\
\text { (75 CE) }\end{array}$ \\
\hline Dons faits & Hors quartier & 0,79 & 0,69 \\
et reçus & Hors village & 0,25 & 0,20 \\
& Parent & 0,50 & $0,17^{\dagger}$ \\
& Ami/connaissance & 0,63 & 0,51 \\
& Intra-ethniques & 0,75 & $0,32^{+\dagger}$ \\
& Interethniques & 1,03 & 0,90 \\
Trocs faits & Intra-quartier & 0,50 & 0,41 \\
et reçus & Hors quartier & 0,04 & $0,23^{\dagger}$ \\
& Hors village & 0,00 & 0,07 \\
& Parent & 0,00 & 0,03 \\
& Ami/connaissance & 0,00 & $0,20^{\dagger}$ \\
& Intra-ethniques & 0,16 & 0,09 \\
Ventes/Achats & 0,04 & $0,32^{\dagger}$ \\
& Interethniques & 0,00 & 0,01 \\
\hline
\end{tabular}

${ }^{\dagger}$ et ${ }^{\dagger \dagger}$ différences entre colonnes significatives respectivement au seuil de 0,10 ou 0,05 . elle-même. Elle confirme également l'existence de réseaux informels d'échange qui assurent jusqu'à $30 \%$ des besoins des villages du Burkina Faso.

Les modes d'échange privilégiés sont le don et le troc. Personne ne peut s'en dispenser sous peine d'être exclu du système global de solidarité. Toutefois, le don étant à l'initiative du donneur, il peut s'avérer insuffisant, et le troc ou l'achat doivent le compléter.

Les échanges se produisent surtout entre voisins de quartier ou de village et entre membres de même famille. Les semences qui sortent du village ou y entrent contribuent à enrichir le portefeuille variétal du village et à maintenir des liens avec les membres de la famille qui ont émigré. Bien qu'essentiel, le lien familial n'est pas exclusif. Dans les villages de Siby, Vélia et Kéra, une part significative des échanges concerne des CE non apparentés et qui ont su instaurer une relation de confiance mutuelle.

Si le réseau traditionnel d'échange de semences apporte une forme de sécurité à ses membres, notre étude montre néanmoins que son efficacité peut être limitée par rapport aux accidents qui affectent sans distinction tous les membres du réseau (climat, épidémies, etc.), ou si le réseau n'entretient pas une certaine diversité variétale. Dans ce cas, le marché est l'ultime recours. C'est ce qui s'est passé à Tiogo et Kéra, où les achats et les ventes ont été importants, en raison d'une production déficitaire liée aux conditions climatiques ou à une forte infestation de Striga hermontica. Les achats correspondant au renouvellement normal des variétés représentent des quantités plus modestes, comme à Siby en 2005.

Comme l'avaient déjà noté Almekinders et al. (1994), nos résultats montrent que, dans des villages à mixité ethnique, les CE échangent préférentiellement au sein de leur groupe ethnique.

La présence de coton dans un village ne bouscule pas les systèmes semenciers informels mais modifie légèrement leur organisation. Le don et le troc représentent plus de $50 \%$ des échanges dans les villages cotonniers de Siby et Kéra comme dans les trois villages sans coton. La présence d'une culture monétarisée n'entraîne pas d'augmentation des échanges marchands. La géographie des échanges reste également similaire.

En revanche, la production de coton crée de nouveaux groupements de professionnels qui complètent ou se substituent partiellement aux réseaux familiaux, sup- 
ports traditionnels de la solidarité. Les échanges entre amis sont ainsi plus nombreux à Siby qu'à Tiogo et même à Kéra en 2004. Les chaînes d'échanges que nous avons reconstituées à Siby confirment que les membres des groupements de producteurs de coton entretiennent des liens entre eux et avec ceux d'autres groupements. L'exemple de Vélia montre toutefois que des réseaux non familiaux peuvent aussi se créer en dehors du coton.

Les limites de cette étude portent sur le lien entre données géographiques et sociales, d'une part, et sur la difficulté d'adopter une définition précise de la famille et de la parentèle, d'autre part. Ainsi, les CE mossis ont plus facilement transgressé les limites de leur quartier ou de leur village lorsque leurs voisins de quartier ou de village étaient également Mossis. Et la notion de parentèle renvoie à une organisation sociale et à des pratiques de solidarité qui correspondent peu à sa traduction occidentale et biologique. Toutefois, ce travail souligne l'intérêt d'une meilleure compréhension du système informel des échanges de semences. S'il suggère que la culture du coton ne déstructure pas les réseaux traditionnels d'échanges, il indique des évolutions qu'une étude approfondie permettrait de mieux comprendre, en intégrant les dimensions économiques, sociologiques et anthropologiques liées à la pratique de cette culture de rente. Il attire également notre attention sur la capacité de résistance, de résilience, et aussi sur la fragilité du système informel. Résistance aux changements engendrés par l'accroissement des rapports médiés par l'argent et l'émergence de nouveaux réseaux sociaux. Résilience dans sa capacité à gérer certaines crises passagères, notamment climatiques, sans bouleversement et sans le secours du système formel (Sperling et Cooper, 2003). Fragilité aussi car les pertes génétiques et les déficits en semences induits par une forte crise ou par une succession de crises, ne peuvent pas être compensés si le système n'est pas alimenté de l'extérieur (McGuire, 2007). Comme Almekinders (2001) ou Lançon et al (2006), nous pensons que l'enjeu est d'élaborer de nouveaux systèmes semenciers qui lient explicitement le formel et l'informel dans la perspective de mieux sécuriser l'approvisionnement en semences des producteurs de sorgho.

\section{Références}

Almekinders CJM, Louwaars NP, De Bruijn GH. Local seed systems and their importance for an improved seed supply in developing countries. Euphytica 1994 ; 78 : 207-16.

Almekinders CJM. Increasing the resilience of farmers'seed system through linkage with the formal sector. In : Sperling $L$, ed. Targeted Seed Aid and Seed-System Interventions: Strengthening Small-Farmer Seed Systems in East and Central Africa. Kampala (Ouganda) Participatory Research and Gender Analysis (PRGA), 2001.
Badstue LB, Bellon MR, Juarez X, Manuel I, Solano AM. In : Social relations and seed transactions among smallscale maize farmers in the central valleys of Oaxaca. Economic Working Paper. Mexico : Centre international pour I'amélioration du maïs et du blé (CIMMYT), 2002.

Lancon J, Hocdé H, Floquet A. Scientifiques et utilisateurs : partenaires pour élaborer de nouveaux systèmes semenciers. In : Lançon J, veaux systemes semenciers. In : Lançon J,
Floquet A, Weltzien E, eds. Partenaires pour construire des projets de sélection participative. Actes de I'atelier-recherche, 14-18 mars 2005, Cotonou, Bénin. Montpellier : Cirad éditions, Inrab, Coopération française, 2006.

McGuire SJ. Vulnerability in farmer seed systems : farmer practices for coping with seed insecurity for sorghum in Eastern Ethiopia. Econ Bot 2007 ; 61 : 211-22.

Mauss M. L'année sociologique, essai sur le don Paris: Presses universitaires de France 1923-1924(réédité dans "Sociologie et Anthropologie ", 1960).

Nagarajan N, Small M. Local seed systems and village-leveldeterminants of millet crop diversity in marginal environment. ETPD discussion paper $2005 ; 135: 1-56$.

Remington T, Maroko J, Walsh S, Omanga $\mathrm{P}$, Charles E. Getting off the seeds-and-tools treadmill with CRS seed vouchers and fairs. Disasters 2002 ; 26 : 316-28.

Sperling L, Cooper D. Understanding seed systems and strengthening seed security. A background paper on effective and sustainable seed relief: a stakeholder workshop, FAO Rome, Italy, 26-28 May, 2003.

Subedi A, Chaudhary P, Baniya BK, et al. Who maintains crop genetic diversity and how? Culture and Agriculture 2003 ; 2 : 41-50.

Tripp R. Seed provision and agricultural development. London : Overseas Development Institute (ODI), 2001.

Van Dusen E. A metapopulation approach to farmer seed systems: Methodology for agricultural biodiversity conservation policy. Bioeconomics $2004 ; 11$ : 1-29. 